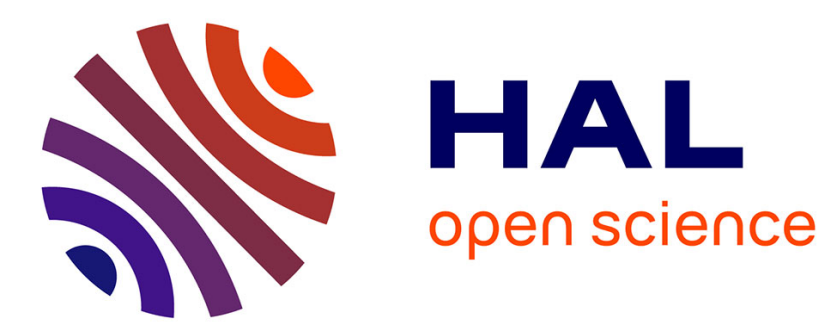

\title{
Modeling the attenuated total reflectance infrared (ATR-FTIR) spectrum of apatite
}

Julie Aufort, Loïc Ségalen, Christel Gervais, Christian Brouder, Etienne Balan

\section{To cite this version:}

Julie Aufort, Loïc Ségalen, Christel Gervais, Christian Brouder, Etienne Balan. Modeling the attenuated total reflectance infrared (ATR-FTIR) spectrum of apatite. Physics and Chemistry of Minerals, 2016, 43 (9), pp.615-626. 10.1007/s00269-016-0821-x . hal-01331139

\section{HAL Id: hal-01331139 \\ https://hal.sorbonne-universite.fr/hal-01331139}

Submitted on 13 Jun 2016

HAL is a multi-disciplinary open access archive for the deposit and dissemination of scientific research documents, whether they are published or not. The documents may come from teaching and research institutions in France or abroad, or from public or private research centers.
L'archive ouverte pluridisciplinaire HAL, est destinée au dépôt et à la diffusion de documents scientifiques de niveau recherche, publiés ou non, émanant des établissements d'enseignement et de recherche français ou étrangers, des laboratoires publics ou privés. 


\title{
Modeling the attenuated total reflectance infrared (ATR-FTIR) spectrum of apatite
}

\author{
Julie Aufort ${ }^{1}$, Loïc Ségalen ${ }^{2}$, Christel Gervais ${ }^{3}$, Christian Brouder $^{1}$, Etienne Balan $^{1}$ \\ ${ }^{1}$ Institut de Minéralogie, de Physique des Matériaux et de Cosmochimie (IMPMC), UMR CNRS 7590, UMR \\ IRD 206, Sorbonne Universités, UPMC Université Paris 6, 4 place Jussieu, 75252 Paris cedex 05, France \\ ${ }^{2}$ ISTEP, Biominéralisations et Environnements Sédimentaires, UMR 7193, Sorbonne Universités, UPMC \\ Université Paris 6, 4 place Jussieu, 75252 Paris cedex 05, France \\ ${ }^{3}$ Laboratoire de Chimie de la Matière Condensée de Paris (LCMCP), Collège de France, Sorbonne Universités, \\ UPMC Université Paris 6, 11 Place Marcelin Berthelot, 75005 Paris, France
}

Corresponding author:

Julie Aufort

Sorbonne Universités - Institut de Minéralogie, de Physique des Matériaux et de Cosmochimie (IMPMC), UMR

CNRS 7590, UMR IRD 206, UPMC Université Paris 6, 4 place Jussieu, 75252 Paris cedex 05, France

Email: Julie.Aufort@impmc.upmc.fr 


\begin{abstract}
Attenuated Total Reflectance (ATR) infrared spectra were measured on a synthetic and a natural fluorapatite sample. A modeling approach based on the computation of the Fresnel reflection coefficient between the ATR crystal and the powder sample was used to analyze the line shape of the spectra. The dielectric properties of the samples were related to those of pure fluorapatite using an effective medium approach, based on Maxwell-Garnett and Bruggeman models. The Bruggeman effective medium model leads to a very good agreement with the experimental data recorded on the synthetic fluorapatite sample. The poorer agreement observed on the natural sample suggests a more significant heterogeneity of the sample at a characteristic length scale larger than the mid-infrared characteristic wavelength, i.e. about 10 micrometers. The results demonstrate the prominent role of macroscopic electrostatic effects over fine details of the microscopic structure in determining the line shape of strong ATR bands.
\end{abstract}




\section{Introduction}

Hard-mode infrared spectroscopy provides a detailed information on the short-range order in minerals (Salje and Bismayer 1997; Salje et al. 2000). It is a particularly relevant technique to investigate a variety of phenomena leading to structural heterogeneity, including the incorporation of molecular impurities (Yi et al. 2014), exsolution processes (Zhang et al. 1996), stacking variations in layered structures (Balan et al. 2010), phase transitions (Salje and Yagil 1996) and cationic ordering (Meyer et al. 2002).

However, the infrared spectra of minerals not only depend on their intrinsic vibrational properties, but are also a function of the sample micro-structure and experimental geometry. In many cases, the spectra are recorded on powder samples and the interplay of the various parameters involved may impede their straightforward interpretation in terms of microscopic structural parameters. This difficulty is particularly apparent when comparing absorption spectra recorded using the traditional pellet method with those obtained using the more recently developed attenuated total reflectance (ATR) infrared spectroscopy. In the first case, the sample consists of particles of the studied mineral diluted in a non-absorbing matrix, usually $\mathrm{KBr}$, and the spectrum is recorded in a transmission geometry. In the second case, the absorption spectrum is recorded in a reflection geometry at the interface between the packed mineral powder and a highly refractive material. ATR thus appears as a very easy experimental procedure which facilitates the acquisition of "absorption-like" infrared spectra by avoiding the multi-step preparation of dry and well-dispersed sample pellets. In many circumstances, it is the only method available to record the infrared spectrum of a given sample. ATR measurements can also be used over a wide frequency range, as attested by measurement of electronic excitations in the near-infrared region (Salje and Güttler 1984). 
However, the differences in sample nature and experimental configuration lead to significant differences in the signals recorded in transmission or ATR geometry.

In the present study, we adopt a modeling approach to investigate the line shape variations observed in the ATR infrared spectra of selected samples of fluorapatite. Apatite is a widespread calcium phosphate mineral with nominal composition $\mathrm{Ca}_{10}\left(\mathrm{PO}_{4}\right)_{6}(\mathrm{OH}, \mathrm{F}, \mathrm{Cl})_{2}$. It occurs in many different geological contexts. For example, world phosphorus resources mainly consist of sedimentary deposits of carbonate-bearing fluorapatite (Knudsen and Gunter 2002), while carbonate-bearing hydroxyapatite is the major inorganic component of vertebrate skeletons (e.g. Elliott 2002; Reynard and Balter 2014). The flexibility of the apatite structure enables significant chemical variations, among which carbonate group incorporation is of peculiar importance (e.g. Leventouri et al. 2000; Pan and Fleet 2002; Antonakos et al. 2007). Infrared spectroscopy of apatite usually provides two different types of information. The crystalline quality of the samples is assessed from the width of absorption bands due to phosphate vibrational modes. This information is usually obtained from relative spectral intensities in the range of $\mathrm{PO}_{4}$ bending vibrational modes $\left(v_{4} \mathrm{PO}_{4}\right)$ and reported as an empirical "crystallinity" index (e.g. Shemesh 1990; Weiner and Bar-Yosef 1990; Surovell and Stiner 2001; Pucéat et al. 2004; Trueman et al. 2008; Roche et al. 2010). The other type of information is related to the concentration and location of molecular species, such as carbonate groups, that are detected by specific vibrational bands (e.g. Michel et al. 1995; Fleet and Liu 2008; Fleet 2009; Yi et al. 2014; Chevrinais et al. 2016). Although the majority of infrared spectroscopic studies of apatite were performed using transmission experiments, recent investigations have used ATR spectroscopy to unravel the modification of biologic apatite during fossilization (e.g. Stathopoulou et al. 2008; Lebon et al. 2014, Lang et al. 2016) and burning (Thompson et al. 2009). Systematic comparison between transmission and ATR 
spectra have revealed small but significant differences in the corresponding "crystallinity" index and carbonate to phosphate ratio (Thompson et al. 2009; Beasley et al. 2014).

Our modeling strategy is similar to that previously used by Balan et al. (2002) to model the ATR-FTIR spectrum of aggregates of chrysotile nanotubes. It combines the computation of the frequency-dependent reflection coefficient at the interface between the ATR crystal and the sample, with an effective medium modeling of the dielectric properties of the composite powder sample. The obtained results make it possible to discuss the respective role of the various physical parameters determining the shape of ATR spectrum and to identify the most appropriate effective medium model that accounts for the properties of apatite powders.

\section{Samples and methods}

Two reference apatite samples have been investigated. The first one is a pure fluorapatite sample containing trace amounts of cadmium $\left(\mathrm{Cd}_{0.005} \mathrm{Ca}_{9.995}\left(\mathrm{PO}_{4}\right)_{6}(\mathrm{~F}, \mathrm{OH})_{2}\right)$ and synthesized at high temperature (Nounah and Lacout 1993). The transmission powder infrared spectrum of this sample has been investigated in details in Balan et al. (2011). The small linewidth $\left(\sim 4 \mathrm{~cm}^{-1}\right)$ of the weak $v_{1} \mathrm{PO}_{4}$ stretching band measured at low temperature $(10 \mathrm{~K})$ attests to the very high crystalline quality of this sample. Scanning electron microscope (SEM) observations display a significant porosity and sub-micrometric coalesced particles with a rounded shape (Fig. 1). The second one is a natural sample of carbonate-fluorapatite, originating from the sedimentary phosphate deposit of Taiba (Senegal) (Morin et al. 2002). It contains $90 \mathrm{wt} \%$ of highly crystalline fluorapatite with a few associated mineral impurities ( $\sim 7 \mathrm{wt} \%$ quartz, $\sim 2 \mathrm{wt} \%$ crandallite, $<1 \mathrm{wt} \%$ goethite, $<1 \mathrm{wt} \%$ dioctahedral smectite). This sample has been previously investigated using transmission powder infrared spectroscopy and 
solid-state nuclear magnetic resonance by Yi et al. (2013). Importantly, the line width of the $v_{1} \mathrm{PO}_{4}$ stretching band is similar to that observed on the synthetic sample, indicating that this natural sample is also very well ordered despite the occurrence of substituted structural carbonate groups. SEM observations reveal that it is mostly composed of aggregated submicrometric prisms of apatite (Fig. 1). For this sample, ATR spectra have been recorded on the as-received sample and after hand-grinding in ethanol during 15 and 60 minutes.

Scanning electron microscope (SEM) observations were performed using a Zeiss ultra 55 SEM operating in the secondary electron mode with a $2 \mathrm{kV}$ accelerating tension and a $2.5 \mathrm{~mm}$ working distance.

The ATR-FTIR spectra have been recorded using a Quest ATR device (Specac) and a Nicolet 6700 FTIR spectrometer equipped with an Ever-Glo source, KBr beamsplitter and DTGS-KBr detector. Pure powder samples have been packed at the surface of the ATR crystal and the spectra have been recorded between 400 and $4000 \mathrm{~cm}^{-1}$ by averaging 200 scans with a resolution of $1 \mathrm{~cm}^{-1}$. For each sample, two different spectra have been recorded using a diamond or a Ge ATR crystal. The ATR spectra are reported in absorbance units.

\section{Modeling strategy}

The modeling strategy is closely related to that successfully used to model the ATR spectrum of a pure chrysotile aggregate by Balan et al. (2002).

The theoretical unpolarized ATR spectrum is obtained by computing the complex Fresnel reflection coefficient for s- and p- polarized waves and an incidence angle of $45^{\circ}$ at a perfectly plane interface between the ATR crystal and the sample (Born and Wolf 1980). The ATR crystal is defined as a homogeneous medium of constant real refractive index (4 for Ge, 
2.4 for diamond); whereas the sample is defined as an infinite homogeneous medium characterized by a frequency-dependent dielectric function.

The porous powder sample corresponds to a binary composite made of the substance of interest, i.e. apatite, and air (here approximated by a homogeneous medium with a unity refractive index). Apatite displaying a crystalline structure with hexagonal symmetry, its dielectric properties are described by a diagonal uniaxial dielectric tensor with a parallel and two identical perpendicular components, noted $\varepsilon_{\|}$and $\varepsilon_{\perp}$, respectively. Numerous models exist that provide the approximate dielectric function of a composite sample from its individual constituents in a quasi-static approach (e.g. Sihvola and Kong 1988; Spanier and Herman 2000; Kendrick and Burnett 2016). They neglect scattering losses and can be straightforwardly extended to frequency-dependent dielectric properties when the characteristic size of individual particles is much smaller than the wavelength of the electromagnetic radiation, a condition usually approximately satisfied in mid-infrared spectroscopy $\left(\lambda_{\mathrm{IR}}>10 \mu \mathrm{m}\right)$. One of the simplest model (here referred to as MG model) was proposed by J.C. Maxwell Garnett (Garnett 1904), in which the particles of one of the constituents are considered as embedded in a homogeneous medium, corresponding to the other constituent for binary samples. In the MG model, the two constituents are therefore not treated in a symmetrically equivalent way. The MG model is closely related to the ClausiusMossotti derivation of the dielectric constant of molecular substances from the polarizability of molecules. For a uniform orientation of particles, the dielectric properties of the composite sample are isotropic. For spherical particles, the $\mathrm{MG}$ effective dielectric function $\varepsilon_{\mathrm{MG}}$ of a uniaxial substance embedded in an isotropic matrix is given by the following expression (Levy and Stroud 1997):

$$
\varepsilon_{M G}=\varepsilon_{h}+3 f \varepsilon_{h} \frac{\left(\varepsilon_{\perp}+2 \varepsilon_{h}\right)\left(\varepsilon_{/ /}-\varepsilon_{h}\right)-2 \varepsilon_{h}\left(\varepsilon_{/ /}-\varepsilon_{\perp}\right)}{(1-f)\left(\varepsilon_{\perp}+2 \varepsilon_{h}\right)\left(\varepsilon_{/ /}+2 \varepsilon_{h}\right)+f \varepsilon_{h}\left(\varepsilon_{\perp}+2 \varepsilon_{/ /}+6 \varepsilon_{h}\right)}
$$


where $f$ is the volume fraction occupied by the particles and $\varepsilon_{h}$ is the dielectric constant of the host matrix. The MG model is well suited to describe the dielectric properties of a sample in which a low amount of one of the constituents is diluted in a non-absorbing matrix, such as the $\mathrm{KBr}$ pellets used for traditional transmission experiments. Note however that the $\mathrm{MG}$ model leads to average properties of the pure material for $f \rightarrow 1$. It also provides physically meaningful results when the sample micro-structure ensures that the dipolar interactions between neighboring particles cancel out, even at intermediate volume fractions. For example, this was the case of the closely packed nanotube aggregates corresponding to the chrysotile sample investigated by Balan et al. (2002). However, for arbitrary micro-structures and intermediate volume fractions, the particles of one of the constituents cannot be considered as fully isolated and embedded in a homogeneous matrix of the other constituent. Correspondingly, an alternative model was used in which each individual particle of a mixture is considered as embedded in an effective medium (Bruggeman 1935, Landauer 1978). At variance with the MG model, all constituents are treated in a symmetrically equivalent way. In this model, the effective dielectric properties of a binary sample consisting in a mixture of an isotropic phase and ellipsoidal particles of a uniaxial phase are defined by the selfconsistent relation (Walker and Scharnberg 1990; Sushko 2009):

$$
f\left\lfloor 2 \frac{\left(1-g_{/ /}\right)\left(\varepsilon_{B r}-\varepsilon_{\perp}\right)}{\left(1+g_{/ /}\right) \varepsilon_{B r}+\left(1-g_{/ /}\right) \varepsilon_{\perp}}+\frac{g_{/ /}\left(\varepsilon_{B r}-\varepsilon_{/ /}\right)}{\left(1-g_{/ /}\right) \varepsilon_{B r}+g_{/ /} \varepsilon_{/ /}}\right\rfloor+3(1-f) \frac{\varepsilon_{B r}-\varepsilon_{h}}{2 \varepsilon_{B r}+\varepsilon_{h}}=0
$$

In this expression, $\mathrm{g}_{/ /}$is the depolarization factor along the particle revolution axis and (1$\mathrm{g}_{/ /} / 2$ corresponds to the depolarization factor in perpendicular directions (for spherical particles, $\left.\mathrm{g}_{/ /}=1 / 3\right)$. The corresponding polynomial equation displays three complex solutions 
among which that with a positive imaginary part has to be selected. A detailed expression of the polynomial coefficients and solutions is given in the annexes.

The uniaxial frequency-dependent dielectric properties of fluorapatite have been modeled with a Drude-Lorentz expression of the coefficients of its diagonal dielectric tensor:

$$
\varepsilon_{\alpha}(\omega)=\varepsilon(\infty)+\sum_{i} \frac{A_{i, \alpha}^{2}}{\left(\omega_{i}^{2}-\omega^{2}-i \omega \Gamma_{i}\right)}
$$

where $\alpha$ refers to the Cartesian axis, $\varepsilon(\infty)=2.65$ is the electronic dielectric tensor of apatite, here considered to be real, constant and isotropic, $\omega_{\mathrm{i}}$ are the vibrational transverse optical (TO) frequencies of IR-active modes, $A_{i, \alpha}{ }^{2}$ are effective mode oscillator strengths, and $\Gamma_{\mathrm{i}}$ is a damping parameter. The frequencies of vibrational modes have been experimentally determined on fluorapatite single-crystals in previous works (e.g. Klee 1970, Adams and Gardner 1974, Leroy et al. 2000). As slight differences occur between the previous experimental determinations, the frequencies used in the present work are listed in Table 1. The mode oscillator strengths used in the present work are those previously obtained by Balan et al. (2011) on fluorapatite using first-principles quantum mechanical calculations. The damping parameter $\Gamma_{\mathrm{i}}$ describes the homogeneous broadening of vibrational bands and has been arbitrarily sets to $8 \mathrm{~cm}^{-1}$ for all the modes.

\section{Results}

\section{a- Comparative properties of the MG and Bruggeman effective models}


As anticipated in their presentation, the two effective medium models used in the present work display very distinct properties as a function of the volume fraction of the constituents. This is particularly evident when considering a model isotropic substance displaying a single resonance in its frequency-dependent complex dielectric function $\varepsilon_{\mathrm{S}}=\varepsilon_{\mathrm{S}}{ }^{\prime}$ $+\mathrm{i} \varepsilon_{\mathrm{S}} "$ (Figure 2). In the limit of a vanishingly small damping coefficient, the maximum of $\varepsilon_{S} "$ defines the transverse optical (TO) frequency; whereas the longitudinal optical (LO) frequency is defined by $\left|\varepsilon_{S}\right|=0$. The second medium of the binary composite is characterized by its dielectric constant $\varepsilon_{\mathrm{h}}$. The relation (1) for the isotropic MG model becomes:

$$
\frac{\varepsilon_{M G}-\varepsilon_{h}}{\varepsilon_{M G}+2 \varepsilon_{h}}=f \frac{\varepsilon_{S}-\varepsilon_{h}}{\varepsilon_{S}+2 \varepsilon_{h}}
$$

The MG dielectric function (Figure 2) still presents a single resonance, which progressively shifts toward the resonance frequency of the pure material when the volume fraction increases. In the limit of low volume fraction and vanishingly small damping parameter, the resonance occurs when $\varepsilon_{S}^{\prime}=2 \varepsilon_{\mathrm{h}}$. For this peculiar condition, the real part of the denominator in Eq. 4 cancels out and the resonance coincides with the surface mode of the isolated sphere. The intensity of the resonance is not linearly related to the volume fraction of the composite but displays a stronger than linear decrease when the volume fraction decreases. Note that an alternative cavity-Maxwell-Garnett model (cavity-MG) can be built by considering air cavities embedded in an isotropic solid. In this case (not shown), two discrete resonances are observed at the TO and LO frequencies of the pure bulk substance.

The symmetric Bruggeman model leads to the following isotropic relation:

$$
(1-f) \frac{\varepsilon_{h}-\varepsilon_{B r}}{\varepsilon_{h}+2 \varepsilon_{B r}}+f \frac{\varepsilon_{S}-\varepsilon_{B r}}{\varepsilon_{S}+2 \varepsilon_{B r}}=0
$$


In the limit cases $f \rightarrow 0$ or $f \rightarrow 1$, the Bruggeman dielectric function is identical to the MG one, with a single resonance at the characteristic frequency of the isolated sphere or of the pure bulk material, respectively. In contrast, the line shape of the imaginary part of the dielectric function at intermediate volume fractions significantly broadens and becomes asymmetric, instead of displaying a single resonance at intermediate frequencies. For volume fractions above $1 / 3$, the lines extend between the $\mathrm{TO}$ and $\mathrm{LO}$ frequencies with a relatively higher intensity on the TO frequency side.

These peculiar shape and dependence on volume fraction can be understood by analyzing the mathematical properties of the model. More specifically, the Bruggeman dielectric function has the properties of a Herglotz function, i.e. it is analytic and maps the upper half complex plane $\left(\left(\varepsilon_{\mathrm{S}} / \varepsilon_{\mathrm{h}}\right) "\right.$ is positive) to itself ( $\varepsilon_{\mathrm{Br}}{ }^{\prime \prime}$ is also positive) (Cherkaeva and Golden 1998). In the present case, it can be shown that the imaginary part of the Bruggeman dielectric function consists in the sum of two terms (see Annexes). One is proportional to ( $3 f$ 1) $\varepsilon_{S} "$ and corresponds to the resonance at the TO frequency observed for $f>1 / 3$. The second one mixes the imaginary and real parts of the dielectric function of the material and leads to the broad feature which extends to the LO frequency. For $f<1 / 3,(3 f-1) \varepsilon_{\mathrm{S}}$ " is negative. As the imaginary part of the Bruggeman dielectric function is positive, this term cancels out with the second one. This explains the significant change in the shape of the Bruggeman dielectric function at $f=1 / 3$. This volume fraction coincides with the percolation threshold of the symmetric Bruggeman model. For conducting particles inserted in a dielectric matrix, such as cermet compounds, it corresponds to the volume fraction above which the sample properties change from the insulating to the conducting regime (e.g. Gadenne et al. 1989).

\section{b- Experimental ATR spectra of apatite}


The ATR spectrum of the synthetic fluorapatite sample (Fig. 3), reported in absorbance units, displays the usual absorption bands ascribed to the vibrational modes of phosphates groups. The bands associated with the $v_{3} \mathrm{PO}_{4}$ vibrations (anti-symmetric stretch) are observed between 1000 and $1150 \mathrm{~cm}^{-1}$. The weak and narrower $v_{1} \mathrm{PO}_{4}$ band (symmetric stretch) is observed at $\sim 966 \mathrm{~cm}^{-1}$. The bands associated with the $v_{4} \mathrm{PO}_{4}$ vibrations (bending modes) are observed between 540 and $620 \mathrm{~cm}^{-1}$. As expected, the ATR spectra depend on the refractive index of the ATR crystal and significant variations are observed between Ge and diamond ATR spectra, with a much lower intensity for the most refractive material (here, Ge with $\mathrm{n}_{\mathrm{Ge}}=4$ ). ATR spectra also differ from the transmission spectrum measured and modeled by Balan et al. (2011) using the traditional method i.e. dilution of apatite particles in a $\mathrm{KBr}$ matrix (Fig. 3). These variations not only consist in changes in spectral intensities but also affect the position and the width of the bands. Interestingly, the line shapes are significantly asymmetric. In the Ge spectrum, the band at $1095 \mathrm{~cm}^{-1}$ extends to $\sim 1130 \mathrm{~cm}^{-1}$, whereas the intense $v_{3} \mathrm{PO}_{4}$ band at $1030 \mathrm{~cm}^{-1}$ extends to $\sim 1090 \mathrm{~cm}^{-1}$. A significant asymmetry is also observed on the $v_{4} \mathrm{PO}_{4}$ band at $\sim 600 \mathrm{~cm}^{-1}$. In the diamond ATR spectrum, the intense $v_{3} \mathrm{PO}_{4}$ band appears broadened toward lower wavenumbers and its maximum is shifted to $1017 \mathrm{~cm}^{-1}$.

The ATR spectrum of the natural carbonate-fluorapatite displays similar features (Fig. 4). Additional small bands related to structural carbonates $\left(v_{2} \mathrm{CO}_{3}\right.$ bending mode at $\sim 865 \mathrm{~cm}^{-}$ $\left.{ }^{1}\right)$ and associated quartz impurities (462, 780 and $799 \mathrm{~cm}^{-1}$ ) are observed. The Ge and diamond ATR spectra of the carbonate-fluorapatite are significantly less intense than the respective spectra of synthetic fluorapatite. Sample grinding tends to increase the spectrum intensity. However, the dependence of the Ge and diamond spectrum as a function of grinding time is different. For diamond ATR, a moderate grinding provides a significant recovery of spectral intensity, which is not improved by further grinding; whereas an increase of the Ge ATR intensity is only observed for the longest grinding time (60 min). This observation can 
be linked to a larger penetration depth for the diamond ATR than for the more refractive Ge one. The strong bands are also broader than in the pure fluorapatite spectrum. In the diamond ATR spectrum, the $v_{3} \mathrm{PO}_{4}$ band extends from 900 to $1100 \mathrm{~cm}^{-1}$. The overlap with the weak band related to the $v_{1} \mathrm{PO}_{4}$ mode leads to a very asymmetric narrow resonance. Its shape shares some similarity with the derivative of a Lorentzian function, suggesting that the real part of the dielectric function related to this resonance significantly contributes to the spectrum. In contrast, this resonance appears with a standard peak shape in the Ge spectrum, in which $v_{1}$ and $v_{3} \mathrm{PO}_{4}$ bands do not overlap.

\section{c- Theoretical ATR-FTIR spectra of apatite}

Starting from a single dielectric function for pure fluorapatite, defined by the parameters specified in Table 1, and for a given effective medium model, the theoretical Ge and diamond ATR spectra are obtained by varying the dielectric constant of the ATR crystal. In this case, the only free parameter that can be used to optimize the agreement between the model ATR spectra and their experimental counterparts is the volume fraction. For the synthetic sample, a good agreement is achieved by using a fraction of 0.5 (Fig. 3) which is reasonable for an ordinary powder sample. For comparison, the ratio of the apparent density of quartz sand to that of pure quartz is 0.577 (Bisal and Hinman 1972).

For the two effective medium models, the shape of the theoretical Ge ATR spectra is very close to that of the imaginary part of the corresponding dielectric functions. The most significant variation are minor shifts $\left(<4 \mathrm{~cm}^{-1}\right)$ of the most intense $v_{3} \mathrm{PO}_{4}$ band toward lower wavenumbers. It is noteworthy that the intensity of the modeled ATR spectra compares well with the raw experimental measurements. As anticipated, the MG model leads to a series of narrow peaks related to the various resonances of the apatite dielectric tensor, accounting 
poorly for the experimental asymmetric line shapes. In contrast, the specific line shapes observed for intermediate volume fractions in the Bruggeman model compare well with their experimental counterparts (Fig. 3). More specifically, they reproduce the peak maximum at low frequency, corresponding to the TO frequencies of the apatite vibrational modes, and the broad absorption feature observed on the high-frequency side. As shown in the analysis of the Bruggeman model, this feature extends up to the LO frequency of apatite vibrational modes. In the $v_{4} \mathrm{PO}_{4}$ range, the Bruggeman model also reproduces the asymmetric line shape of the band at $600 \mathrm{~cm}^{-1}$ and the line splitting observed at 564 and $578 \mathrm{~cm}^{-1}$. Considering the diamond ATR spectrum, its shape differs from that of the imaginary part of dielectric functions by the fact that the most intense $v_{3} \mathrm{PO}_{4}$ band is broadened toward the lower wavenumbers for the two effective medium models. This effect on the line shape is therefore specifically ascribed to the ATR experimental configuration. As for the Ge ATR spectrum, the best agreement on the line positions and spectral shape is obtained by using the Bruggeman dielectric model, both in the $v_{3} \mathrm{PO}_{4}$ and $v_{4} \mathrm{PO}_{4}$ ranges. The experimentally observed position of the bands, and more importantly, the relative changes between the spectra and the relative shifts with respect to the resonances of the dielectric tensor of pure bulk apatite are well-reproduced by the model. The slight but systematic under-estimation of the position of the weak $v_{1} \mathrm{PO}_{4}$ band (by $4 \mathrm{~cm}^{-1}$ ) suggests that the TO frequency used to model the low-frequency dielectric tensor of apatite (Table 1) was slightly underestimated. Note that attempts using the cavity-MG model did not provide a better modeling of the observed peculiar line shapes than the standard MG model. Indeed, this model also leads to a series of discrete peaks instead of the broad features extending between TO and LO frequencies for intermediate volume fractions.

The ATR spectrum of the natural sample of carbonate-fluorapatite was modeled using the same apatite dielectric tensor as for the synthetic one (Fig. 5). No attempt has been made 
to take into account the small spectroscopic signal related to the structural carbonate ions. Importantly, the line width was not modified, which is consistent with the small $v_{1} \mathrm{PO}_{4}$ linewidth measured in previous transmission IR spectroscopy studies (Balan et al. 2011; Yi et al. 2013). This small linewidth attests to the weakness of the inhomogeneous broadening related to microscopic disorder parameters. A moderate increase in the apatite volume fraction significantly increases the asymmetric broadening of the intense $v_{3} \mathrm{PO}_{4}$ band in the diamond ATR spectrum. It overlaps with the narrow $v_{1} \mathrm{PO}_{4}$ band, leading to a shape similar to the derivative of a Lorentzian, consistent with the experimental observations. However, the theoretical ATR spectra do not account for the significant decrease in spectral intensity observed from the synthetic to the natural sample. The modeled diamond and Ge spectra consistently overestimate their experimental counterparts by a factor of approximately two (Fig. 5). This points to a failure in the model of the sample effective dielectric properties. A microscopic sample heterogeneity, e.g. related to carbonate substitutions, cannot explain such discrepancy because it is not supported by the observed $v_{1} \mathrm{PO}_{4}$ linewidth. In contrast, heterogeneities occurring at a length scale larger than the characteristic length scale of the spectroscopic measurements, typically the IR wavelength, could account for the observed discrepancy. We particularly suspect spatial fluctuations in the solid/air fraction to account for the observed discrepancy.

\section{Concluding remarks}

The present modeling of apatite ATR spectra demonstrates the prominent role of macroscopic electrostatic effects over fine details of the microscopic structure in the line shape of strong ATR bands. A relatively simple effective medium modeling of the dielectric properties of the sample, based on the Bruggeman approximation, already provides important 
insights into these respective contributions. It points to the key role of the volume fraction of the constituents, which is the only free parameter of the model. More sophisticated models would most probably improve the agreement between theory and experiment (e.g. Yagil et al. 1995; Spanier and Herman 2000) but at the price of a corresponding increase in the number of free parameters.

As previously discussed in Balan et al. (2011), macroscopic electrostatic effects have a weaker influence on the shape and position of the weak absorption bands of apatite. These bands, although often neglected and sometimes more difficult to record, are expected to provide a better insight into the short-range order in crystal structures. However, the present modeling indicates that their overlap with stronger resonances in the diamond ATR spectrum significantly affects their shape by mixing the real and imaginary parts of the dielectric function.

Finally, it appears that the relation between ATR and transmission spectra is not straightforward even though commercial softwares provide ATR correction algorithms to facilitate the comparison. The different composite nature (substance/air vs substance/KBr) and more importantly the different dilution state (pure powder vs isolated particles) of the measured samples affect their macroscopic electrostatic properties and result in changes in the position, shape and relative intensity of strong absorption bands. This can significantly bias the comparison between ATR and transmission spectra despite the fact that the analyzed bulk substance is characterized by a single dielectric function. Importantly, the differences observed in the analyzed samples are explained by the long-range character of electrostatic interactions affecting (sub-)micronic particles. They differ in essence from potential surfaceinduced relaxation effects which occur at the atomic scale and can become dominant in nanometer-sized particles (Waychunas and Zhang 2008). 


\section{Acknowledgments}

We thank G. Morin for providing us with the fluorapatite samples. Support by M. Guillaumet, I. Estève and the IMPMC spectroscopy and SEM-FIB facilities is acknowledged. We thank Prof E.K.H. Salje and an anonymous reviewer for thoughtful and constructive reviews of this manuscript. This work was supported by French state funds managed by the ANR within the Investissements d'Avenir programme under reference ANR-11-IDEX-0004-02, and more specifically within the framework of the Cluster of Excellence MATISSE led by Sorbonne Universités. Support from the Convergence Program "Environnements \& Société" of Sorbonne Universités is acknowledged.

\section{References}

Adams DM, Gardner IR (1974) Single-crystal vibrational spectra of apatite, vanadinite and mimetite. J Chem Soc Dalton 14:1505-1509

Antonakos A, Liarokapis E, Leventouri T (2007) Micro-Raman and FTIR studies of synthetic and natural apatites. Biomaterials 28:3043-3054

Balan E, Mauri F, Lemaire C, Brouder C, Guyot F, Saitta AM, Devouard B (2002) Multiple ionic plasmon resonances in naturally-occurring multiwall nanotubes: infrared spectra of chrysotile asbestos. Phys Rev Lett 89:177401

Balan E, Delattre S, Guillaumet M, Salje EKH (2010) Low-temperature infrared spectroscopic study of $\mathrm{OH}$ stretching modes in kaolinite and dickite. Am Mineral 95:12571266

Balan E, Delattre S, Roche D, Segalen L, Morin G, Guillaumet M, Blanchard M, Lazzeri M, Brouder C, Salje EKH (2011) Line-broadening effects in the powder infrared spectrum of apatite. Phys Chem Miner 38:111-122 
Beasley MM, Bartelink EJ, Taylor L, Miller RM (2014) Comparison of transmission FTIR, ATR, and DRIFT spectra: implications for assessment of bone bioapatite diagenesis. J Archaeol Sci 46:16-22

Bisal F, Hinman WC (1972) A method of estimating the apparent density of soil aggregates. Can J Soil Sci 52:513-514

Born M, Wolf E (1980) Principles of Optics (6th edition). Pergamon Press, New York.

Bruggeman DAG (1935) Berechnung verschiedener physikalischer Konstanten von heterogenen Substanzen. I. Dielektrizitätskonstanten und Leitfähigkeiten der Mischkörper aus isotropen Substanzen. Annalen der Physik 416:665-679

Cherkaeva E, Golden KM (1998) Inverse bounds for microstructural parameters of composite media derived from complex permittivity measurements. Wave Random Media 8(4):437-450

Chevrinais M, Balan E, Cloutier R (2016) New insights in the ontogeny and taphonomy of the devonian acanthodian Triazeugacanthus affinis from the Miguasha Fossil-Lagerstätte, Eastern Canada. Minerals 6:1.

Elliott JC (2002) Calcium Phosphate Biominerals. In: M.J. Kohn, J. Rakovan and J.M. Hughes (eds) Phosphates: Geochemical, Geobiological, and Materials Importance, 48, pp. 427-453. Reviews in Mineralogy and Geochemistry, Mineralogical Society of America and Geochemical Society, Chantilly, Virginia

Fleet ME, Liu X (2008) Accommodation of the carbonate ion in fluorapatite synthesized at high pressure. Am Mineral 93:1460-1469

Fleet ME (2009) Infrared spectra of carbonate apatites: v2-region bands. Biomaterials 30:1473-1481

Gadenne M, Lafait J, Gadenne P. (1989) Infrared absorption of Au-A12O3 thin cermet films: experiment, Bruggeman model, far and near the percolation threshold. Physica A 157:400406 
Garnett JCM (1904) Colours in metal glasses and metallic films. Philos Trans Roy Soc A $203: 385-420$

Kendrick J and Burnett AD (2016) PDielec: The calculation of infrared and terahertz absorption for powdered crystals. J Comput Chem 37:1491-1504

Klee WE (1970) The vibrational spectra of the phosphate ions in fluorapatite. Z Kristallogr $131: 95-102$

Knudsen AC, Gunter ME (2002) Sedimentary phosphates - An example: Phosphoria formation, Southern Idaho, U.S.A. In: Kohn MJ, Rakovan J Hughes JM (eds) Phosphates: Geochemical, Geobiological and Materials Importance. Rev Mineral Geochem 48:363-389 Landauer R (1978) Electrical conductivity in inhomogeneous media. AIP Conf Proc 40:2-45 Lang L, Kirsimäe K, Vahur S (2016) Diagenetic fate of bioapatite in linguliform brachiopods: multiple apatite phases in shells of Cambrian lingulate brachiopod Ungula ingrica (Eichwald). Lethaia 49:13-27

Lebon M, Zazzo A, Reiche I (2014) Screening in situ bone and teeth preservation by ATRFTIR mapping. Palaeogeogr, Palaeocl 416:110-119

Leroy G, Leroy N, Penel G, Rey C, Laforgue P, Bres E (2000) Polarized micro-raman study of fluorapatite single crystals. Appl Spectrosc 54(10):1521-1527

Leventouri T, Chakoumakos BC, Moghaddam HY, Perdikatsis V (2000) Powder neutron diffraction studies of a carbonate fluorapatite. J Mater Res 15:511-517

Levy O, Stroud D. (1997) Maxwell Garnett theory for mixtures of anisotropic inclusions: Application to conducting polymers. Phys Rev B 56:8035-8046

Meyer H-W, Carpenter MA, Becerro AI, Seifert F (2002) Hard-mode infrared spectroscopy of perovskites across the $\mathrm{CaTiO}_{3}-\mathrm{SrTiO}_{3}$ solid solution. Am Mineral 87:1291-1296 
Michel V, Ildefonse P, Morin G (1995) Chemical and structural changes in Cervus Elaphus tooth enamels during fossilization (Lazaret cave): a combined IR and XRD Rietveld analysis. Appl Geochem 10:145-159

Morin G, Allard T, Balan E, Ildefonse P, Calas, G (2002) Native $\mathrm{Cd}^{+}$in sedimentary fluorapatite. Eur J Mineral 14:1087-1094

Nounah A, Lacout JL (1993) Thermal behavior of cadmium containing apatites. J Solid State Chem 107:444-451

Pan Y, Fleet M (2002) Compositions of the apatite-group minerals: substitution mechanisms and controlling factors. In: Kohn MJ, Rakovan J, Hughes JM (eds) Phosphates: Geochemical, Geobiological and Materials Importance. Rev Miner Geochem 48:13-49

Pucéat E, Reynard B, Lécuyer C (2004) Can crystallinity be used to determine the degree of chemical alteration of biogenic apatites? Chem Geol 205:83-97

Reynard B, Balter V (2014) Trace elements and their isotopes in bones and teeth: Diet, environments, diagenesis, and dating of archeological and paleontological samples. Palaeogeogr, Palaeocl 416:4-16

Roche D, Segalen L, Balan E, Delattre S (2010) Preservation assessment of Miocene-Pliocene tooth enamel from Tugen Hills (Kenyan Rift Valley) through FTIR, chemical and stableisotope analyses. J Archaeol Sci 37:1690-1699

Salje E, Güttler B (1984) Anderson transition and intermediate polaron formation in $\mathrm{WO}_{3-\mathrm{x}}$ Transport properties and optical absorption Philos Mag B 50:607-620

Salje EKH, Carpenter MA, Malcherek T, Boffa Balaran T (2000) Autocorrelation analysis of infrared spectra from minerals. Eur J Mineral 12:503-519

Salje EKH, Bismayer U (1997) Hard mode spectroscopy: the concept and applications. Phase Transit 63:1-75 
Salje EKH, Yagil Y (1996) Hard mode spectroscopy for the investigation of structural and superconducting phase transitions. J Phys Chem Solids 57:1413-1424

Shemesh A. (1990) Crystallinity and diagenesis of sedimentary apatites. Geochim Cosmochim Ac 54:2433-2438

Shusko MY (2009) Effective permittivity of mixtures of anisotropic particles. J Phys D Appl Phys 42(15):155410

Sihvola AH, Kong JA (1988) Effective permittivity of dielectric mixtures. IEEE T Geosci Remote 26:420-429

Spanier JE, Herman IP (2000) Use of hybrid phenomenological and statistical effectivemedium theories of dielectric functions to model the infrared reflectance of porous SiC films. Phys Rev B 61:10437-10450

Stathopoulou ET, Psycharis V, Chryssikos GD, Gionis V, Theodorou G (2008) Bone diagenesis: New data from infrared spectroscopy and X-ray diffraction. Palaeogeogr, Palaeocl 266:168-174

Surovell TA, Stiner MC (2001) Standardizing infra-red measures of bone mineral crystallinity: an experimental approach. J Archaeol Sci 28:633-642

Thompson TJU, Gauthier M, Islam M (2009) The application of a new method of Fourier Transform Infrared Spectroscopy to the analysis of burned bone. J Archaeol Sci 36:910-914.

Trueman CN, Privat K, Field J (2008) Why do crystallinity values fail to predict the extent of diagenetic alteration of bone mineral? Palaeogeogr, Palaeocl 266:160-167

Walker D, Scharnberg K (1990) Electromagnetic response of high-Tc superconductors. Phys Rev B 42:2211-2221

Waychunas GA, Zhang H (2008) Structure, chemistry and properties of mineral nanoparticles Elements 4:381-387 
Weiner S, Bar-Yosef O (1990) States of preservation of bones from prehistoric sites in the Near East: A survey. J Archaeol Sci 17:187-196

Yagil Y, Baudenbacher F, Zhang M, Birch JR, Kinder H, Salje EKH (1995) Optical properties of $\mathrm{YBa}_{2} \mathrm{Cu}_{3} \mathrm{O}_{7-\mathrm{d}}$ thin films. Phys Rev B 52:15582-15591

Yi H, Balan E, Gervais C, Segalen L, Roche D, Fayon F, Person A, Morin G, Babonneau F (2014) Probing atomic scale transformation of fossil enamel using FTIR and NMR spectroscopy: A case study from the Tugen Hills (Rift Gregory, Kenya). Acta Biomater $10: 3952-3958$

Yi H, Balan E, Gervais C, Segalen L, Fayon F, Roche D, Person A, Morin G, Guillaumet M, Blanchard M, Lazzeri M, Babonneau F (2013) A carbonate-fluoride defect model for carbonate-rich fluorapatite. Am Mineral 98:1066-1069

Zhang M, Wruck B, Graeme Barber A, Salje EKH, Carpenter MA (1996) Phonon spectroscopy on alkali-feldspars: Phase transitions and solid solutions. Am Mineral 81:92-104 


\section{Annexes:}

\section{1- Complex solutions of the uniaxial Bruggeman model}

The Bruggeman relation is (Eq. 2):

$$
f\left\lfloor 2 \frac{\left(1-g_{/ /}\right)\left(\varepsilon_{B r}-\varepsilon_{\perp}\right)}{\left(1+g_{/ /}\right) \varepsilon_{B r}+\left(1-g_{/ /}\right) \varepsilon_{\perp}}+\frac{g_{/ /}\left(\varepsilon_{B r}-\varepsilon_{/ /}\right)}{\left(1-g_{/ /}\right) \varepsilon_{B r}+g_{/ /} \varepsilon_{/ /}}\right\rfloor+3(1-f) \frac{\varepsilon_{B r}-\varepsilon_{h}}{2 \varepsilon_{B r}+\varepsilon_{h}}=0
$$

The solutions of the equation are the roots of a cubic polynomial with complex coefficients:

$a x^{3}+b x^{2}+c x+d=0$

with

$$
\begin{aligned}
& a=-3-f+6 f g_{/ /}+3 g_{/ /}^{2}-9 f g_{/}^{2} \\
& b=3\left(\varepsilon_{h}-\varepsilon_{\perp}\right)+\left(7 \varepsilon_{\perp}-5 \varepsilon_{h}\right) f+\left(6 \varepsilon_{\perp}-3 \varepsilon_{/ /}\right) g_{/ /}+f g_{/ /}\left(3 \varepsilon_{h}+\varepsilon_{/ /}-16 \varepsilon_{\perp}\right)-3 g_{/ /}^{2}\left(\varepsilon_{/ /}+\varepsilon_{\perp}+\varepsilon_{h}\right)+9\left(\varepsilon_{/ /}+\varepsilon_{\perp}\right) f g_{/ /}^{2} \\
& c=\varepsilon_{h} \varepsilon_{\perp}(3-f)+\left(3 \varepsilon_{h} \varepsilon_{/ /}-6 \varepsilon_{h} \varepsilon_{\perp}-3 \varepsilon_{/ /} \varepsilon_{\perp}\right) g_{/ /}+\left(9 \varepsilon_{/ /} \varepsilon_{\perp}+\varepsilon_{h} \varepsilon_{\perp}-4 \varepsilon_{h} \varepsilon_{/ /}\right) f g_{/ /} \\
& +3 g_{/ /}^{2}\left(\varepsilon_{h} \varepsilon_{/ /}+\varepsilon_{h} \varepsilon_{\perp}+\varepsilon_{/ /} \varepsilon_{\perp}\right)-9 \varepsilon_{/ /} \varepsilon_{\perp} f g_{/ /}^{2} \\
& d=3 \varepsilon_{h} \varepsilon_{/ /} \varepsilon_{\perp}\left(g_{/ /}-g_{/ /}^{2}\right)
\end{aligned}
$$

The three roots are:

$$
\begin{aligned}
& x_{1}=-\frac{b}{3 a}-\frac{2^{1 / 3}\left(-b^{2}+3 a c\right)}{3 a\left(-2 b^{3}+9 a b c-27 a^{2} d+\sqrt{-4\left(b^{2}-3 a c\right)^{3}+\left(2 b^{3}-9 a b c+27 a^{2} d\right)^{2}}\right)^{1 / 3}} \\
& +\frac{\left(-2 b^{3}+9 a b c-27 a^{2} d+\sqrt{-4\left(b^{2}-3 a c\right)^{3}+\left(2 b^{3}-9 a b c+27 a^{2} d\right)^{2}}\right)^{1 / 3}}{3 a 2^{1 / 3}} \\
& x_{2}=-\frac{b}{3 a}+\frac{(1+i \sqrt{3})\left(-b^{2}+3 a c\right)}{3 a 2^{2 / 3}\left(-2 b^{3}+9 a b c-27 a^{2} d+\sqrt{-4\left(b^{2}-3 a c\right)^{3}+\left(2 b^{3}-9 a b c+27 a^{2} d\right)^{2}}\right)^{1 / 3}} \\
& -\frac{(1-i \sqrt{3})\left(-2 b^{3}+9 a b c-27 a^{2} d+\sqrt{-4\left(b^{2}-3 a c\right)^{3}+\left(2 b^{3}-9 a b c+27 a^{2} d\right)^{2}}\right)^{1 / 3}}{6 a 2^{1 / 3}} \\
& x_{3}=-\frac{b}{3 a}+\frac{(1-i \sqrt{3})\left(-b^{2}+3 a c\right)}{3 a 2^{2 / 3}\left(-2 b^{3}+9 a b c-27 a^{2} d+\sqrt{-4\left(b^{2}-3 a c\right)^{3}+\left(2 b^{3}-9 a b c+27 a^{2} d\right)^{2}}\right)^{1 / 3}} \\
& -\frac{(1+i \sqrt{3})\left(-2 b^{3}+9 a b c-27 a^{2} d+\sqrt{-4\left(b^{2}-3 a c\right)^{3}+\left(2 b^{3}-9 a b c+27 a^{2} d\right)^{2}}\right)^{1 / 3}}{6 a 2^{1 / 3}}
\end{aligned}
$$

Among these three roots, only that with a positive imaginary part has to be selected. 


\section{2- Some mathematical properties of the Bruggeman model}

The solutions of Eq. 5 are the roots of a quadratic polynomial equation with complex coefficients:

$x^{2}+2 b x+c=0$

with $x=\frac{\varepsilon_{B r}}{\varepsilon_{h}}, b=-\frac{(3 f-1) \frac{\varepsilon_{S}}{\varepsilon_{h}}+(2-3 f)}{4}$ and $c=-\frac{\varepsilon_{S}}{2 \varepsilon_{h}} . \varepsilon_{\mathrm{h}}$ is the dielectric constant of the host and $\varepsilon_{S}=\varepsilon_{S}{ }^{\prime}+\mathrm{i} \varepsilon_{S} "$ is the complex dielectric function of the substance.

The corresponding solutions are: $x_{ \pm}=-b \pm \sqrt{\Delta}$ with $\Delta=b^{2}-c$. We will now show that the imaginary part of the solution $\mathrm{x}_{+}$is positive.

The imaginary part of $\sqrt{\Delta}$ is equal to $\sqrt{\left(|\Delta|-\Delta_{r}\right) / 2}$ where $\Delta=\Delta_{r}+i \Delta_{i}$. The imaginary part of $\mathrm{X}_{+}$is thus:

$$
\operatorname{Im}\left(x_{+}\right)=-b_{i}+\sqrt{\frac{\left(|\Delta|-\Delta_{r}\right)}{2}}
$$

where $b=b_{r}+i b_{i}$

We now show that the absolute value $\left|b_{i}\right|$ is smaller than $\sqrt{\frac{\left(\Delta \mid-\Delta_{r}\right)}{2}}$. This is equivalent to determining that $|\Delta|^{2}-\left(2 b_{i}^{2}+\Delta_{r}\right)^{2}$ is positive. Replacing the coefficients $\Delta$ and $b_{\mathrm{i}}$ by their expressions as a function of the dielectric function of the substance and matrix, we obtain: $|\Delta|^{2}-\left(2 b_{i}^{2}+\Delta_{r}\right)^{2}=\frac{9}{8}\left(\frac{\varepsilon_{S}^{\prime \prime}}{\varepsilon_{h}}\right)^{2} f(1-f)$ which is indeed always positive for $0 \leq f \leq 1$.

As a consequence, $\operatorname{Im}\left(x_{+}\right)$is also positive. This property is even stronger than the Herglotz property because $\varepsilon_{\mathrm{S}}$ " is not assumed to be positive. In particular for $f=0, \operatorname{Im}\left(\mathrm{x}_{+}\right)=$ 
$\left(\varepsilon_{S} "+\left|\varepsilon_{S} "\right|\right) / 2 \geq 0$ even if $\varepsilon_{S} "$ is negative. The condition that $\varepsilon_{h}$ is real and positive thus implies that $\operatorname{Im}\left(\varepsilon_{\mathrm{Br}}\right)$ is positive, for any function $\varepsilon_{\mathrm{S}}$.

As $-b_{i}=(3 f-1) \frac{\varepsilon_{S}^{\prime \prime}}{4 \varepsilon_{h}}$ and $\varepsilon_{\mathrm{S}}^{\prime \prime}>0$, the first term in Eq. A1 is positive when $\mathrm{f}>1 / 3$. This contribution is thus proportional to the dielectric function of the pure bulk substance and always present. For $f<1 / 3$, this contribution becomes negative but since $\operatorname{Im}\left(\mathrm{x}_{+}\right) \geq 0$, this negative contribution is compensated for by the second term in Eq. A1. The $\varepsilon_{\mathrm{S}}$ " peak then disappears completely from the spectrum. Equation A1 has another interesting consequence. In a region where $\left|\Delta_{\mathrm{i}}\right|$ is smaller than $\left|\Delta_{\mathrm{r}}\right|$, there are two regimes: if $\Delta_{\mathrm{r}} \geq 0$, then $|\Delta|-\Delta_{\mathrm{r}} \approx$ $\Delta_{\mathrm{i}}^{2} /\left(2 \Delta_{\mathrm{r}}\right)$ is small and the first term of Eq. A1 is dominant for $f>0.3$; if $\Delta_{\mathrm{r}} \leq 0$, then $|\Delta|-\Delta_{\mathrm{r}} \approx$ $2\left|\Delta_{\mathrm{r}}\right|$ and the deformation of the spectrum due to the second term of Eq. A1 becomes large. In other words, the deformation of the spectrum of apatite for $f>0.3$ occurs when $\Delta$ has a large negative real part.

We now examine the properties of the imaginary part of the solution for $f=0.5$ and $\varepsilon_{\mathrm{h}}=1$, i.e. the parameters used to model the spectrum of synthetic fluorapatite (Fig. 6). In this case, $b^{2}-c=\varepsilon_{S}\left(\varepsilon_{S}+34\right)+1$. For a vanishingly small damping parameter and out of the TO frequency, $\varepsilon_{\mathrm{S}}$ is real: $\varepsilon_{\mathrm{S}} \approx \varepsilon_{\mathrm{S}}$. Therefore, only the second term contributes to the imaginary part of the solution when $b^{2}-c$ is negative. This condition is met for $\varepsilon_{S}{ }^{\prime}$ approximately ranging between -34 and zero, i.e. on the high energy side of the TO frequency and up to about the LO frequency. For $\varepsilon_{S}^{\prime}$ below -34, the imaginary part of $\varepsilon_{S}$ becomes dominant and the spectrum is proportional to the dielectric function of the pure bulk substance. 


\section{Figures:}
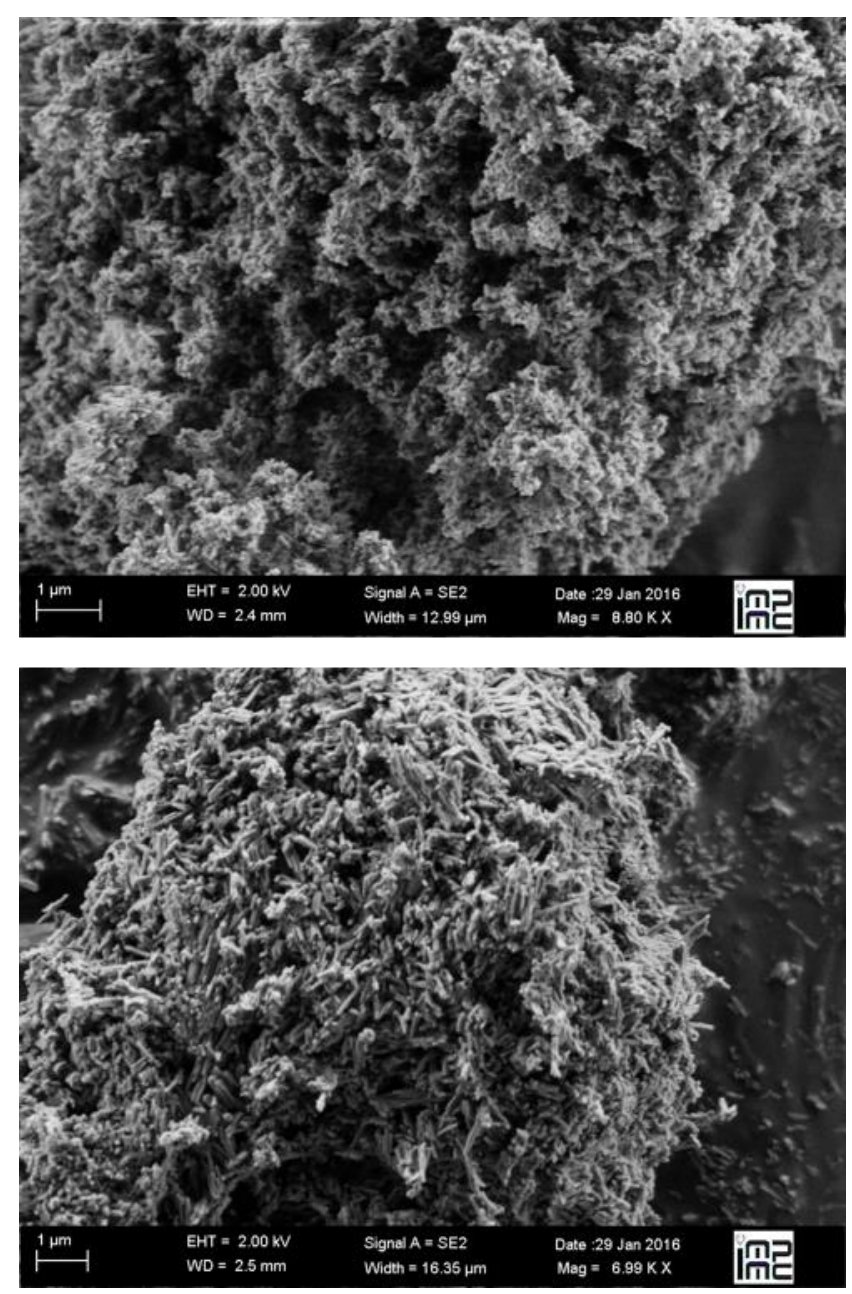

Figure 1: SEM micrographs of synthetic fluorapatite (top) and natural carbonate-fluorapatite (bottom). 

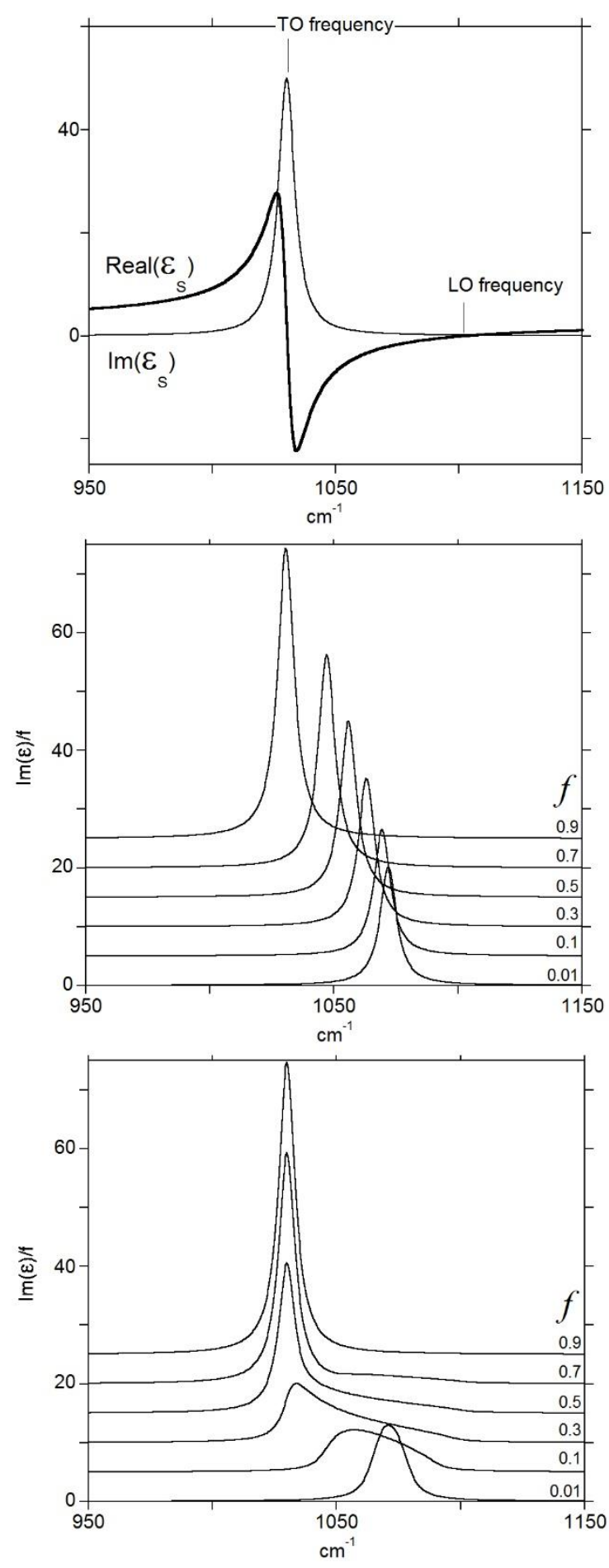

Figure 2: Real and imaginary parts of a model single-resonance dielectric function (top) and corresponding imaginary part of the effective dielectric function in the Maxwell-Garnett (middle) and Bruggeman (bottom) models. The parameters of the model dielectric function (Eq. 3) were $\omega_{0}=1030 \mathrm{~cm}^{-1}, A_{\alpha}=642, \Gamma=8 \mathrm{~cm}^{-1}, \varepsilon(\infty)=2.65$. The effective dielectric functions have been divided by the corresponding volume fraction $f$ value, reported on the right side. 

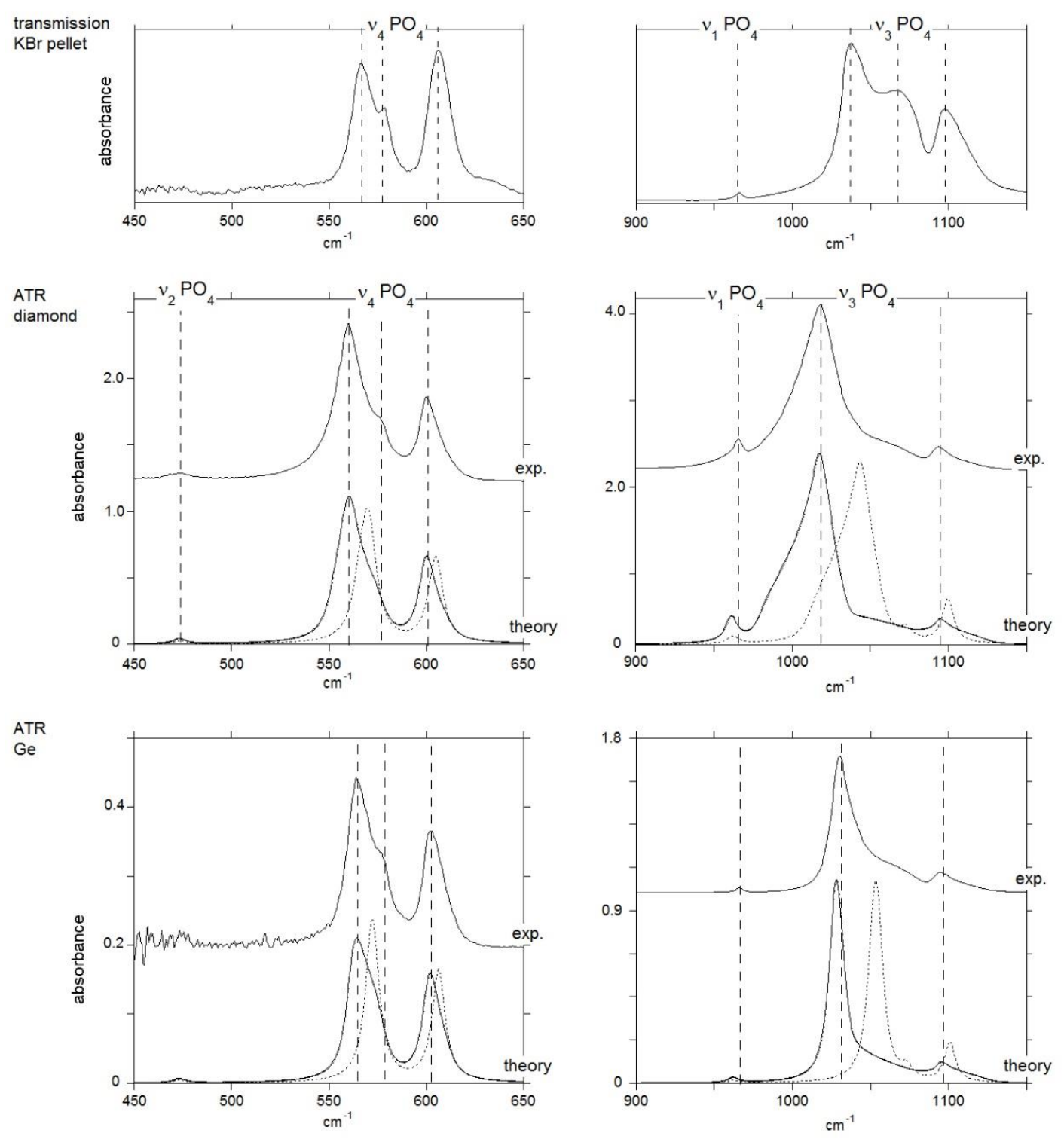

Figure 3: Experimental and modeled diamond (middle) and Ge (bottom) ATR spectra of synthetic fluorapatite. Experimental spectra have been vertically shifted for clarity. MaxwellGarnett and Bruggeman modeled ATR spectra are displayed as dotted and solid lines respectively. The transmission spectrum recorded by Balan et al. (2011) on the same sample after dilution in $\mathrm{KBr}$ is reported for comparison (top). Note the significant changes affecting strong absorption bands. Vertical dotted lines serve as guide to the eye. 

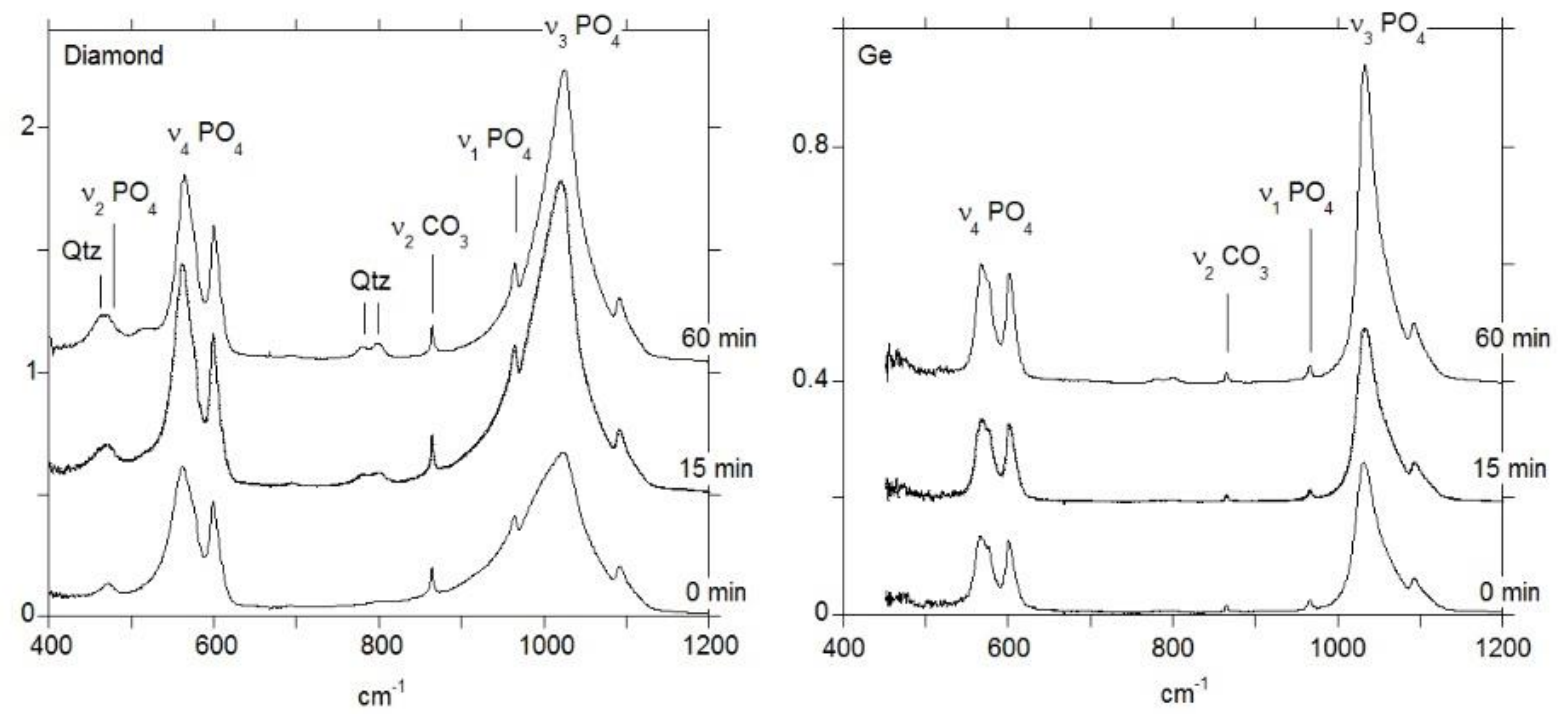

Figure 4: Experimental ATR spectra of natural carbonate-fluorapatite samples. Grinding times are reported on the right side for each spectrum. Grinding time affects the intensity of Ge and diamond spectra differently, suggesting an interplay between the sample spatial heterogeneity and characteristic IR penetration depth in the ATR crystals. 

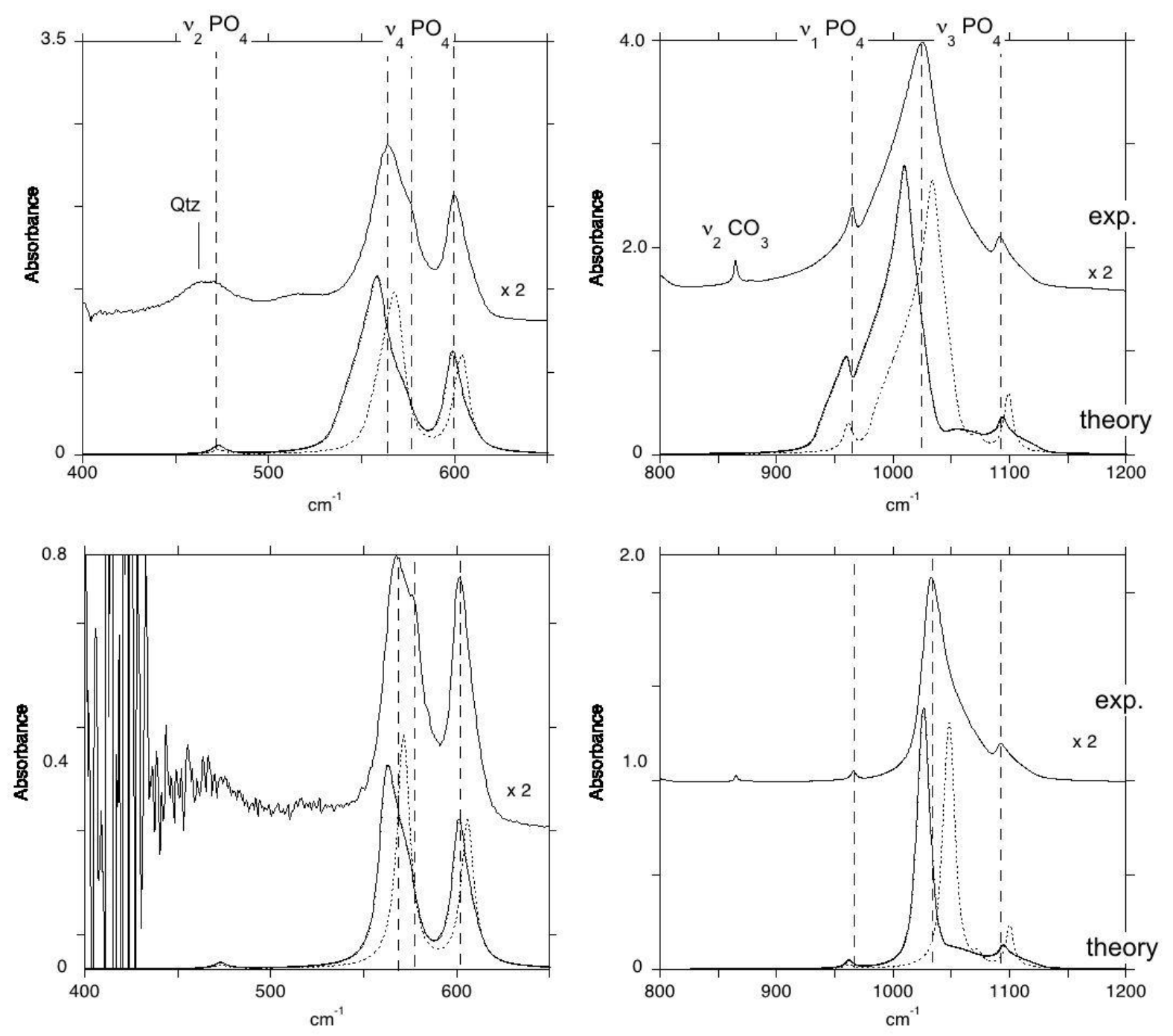

Figure 5: Experimental and modeled diamond (top) and Ge (bottom) ATR spectra of natural carbonate-fluorapatite. Experimental spectra have been vertically shifted for clarity and experimental intensities were multiplied by 2 . Note that the wavenumber ranges slightly differ from those on figure 3. Maxwell-Garnett and Bruggeman modeled ATR spectra are displayed as dotted and solid lines respectively. 


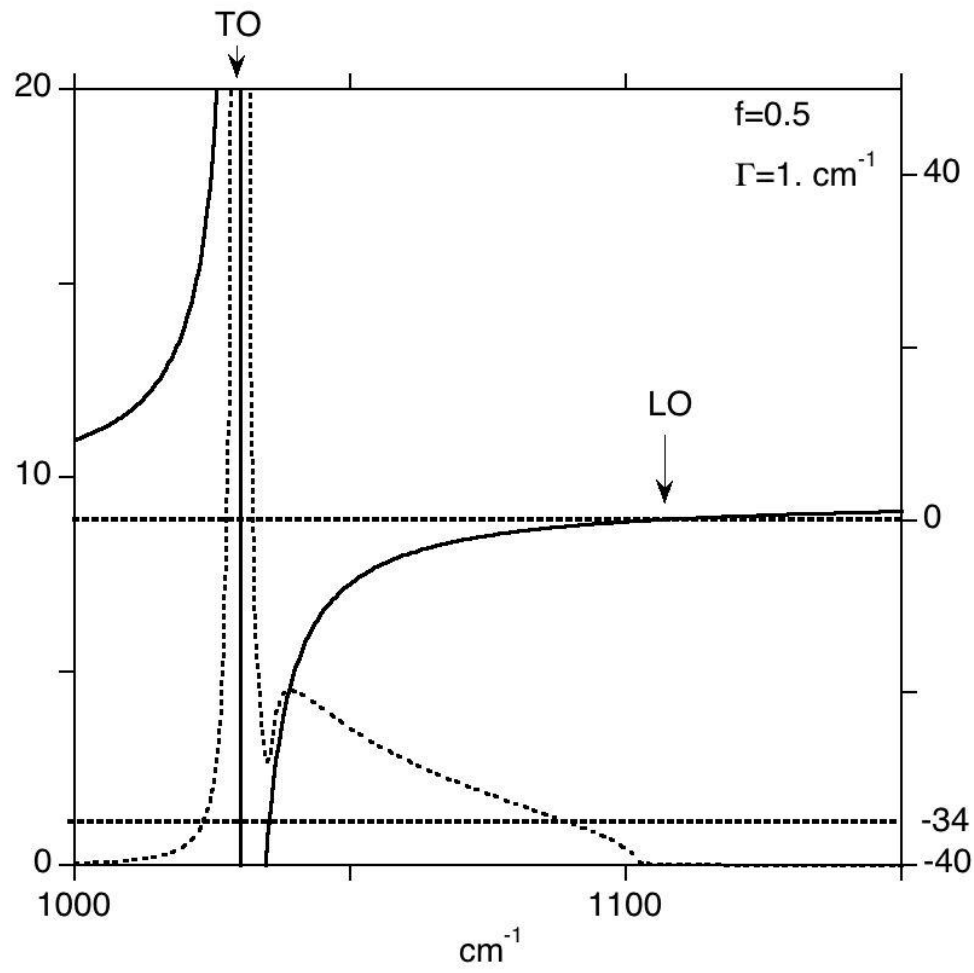

Figure 6: Real part of the model dielectric function (bold line, right axis) and imaginary part of the corresponding effective dielectric function (dotted line, left axis). The small damping parameter $\left(1 \mathrm{~cm}^{-1}\right)$ makes it possible to discriminate the two superposed contributions for a volume fraction of 0.5 . The thin contribution at TO frequency displays an almost Lorentzian shape proportional to the imaginary part of the model dielectric function. In contrast, the broad contributions at higher frequency is observed when the real part of the model dielectric function takes values between $\sim-34$ and $\sim 0$ (LO frequency), indicated by the horizontal dotted lines. 
Table 1: Parameters of the model dielectric tensor of apatite (Eq. 3).

\begin{tabular}{lcccc}
\hline Symmetry & Mode & $\omega_{\mathrm{i}}\left(\mathrm{cm}^{-1}\right)$ & $\mathrm{A}_{\mathrm{i}, \mathrm{a}}(\text { arb. units })^{4}$ & $\Gamma_{\mathrm{i}}\left(\mathrm{cm}^{-1}\right)$ \\
\hline $\mathrm{A}_{\mathrm{u}}$ & $v_{3}$ & $1030^{1}$ & 665 & 8 \\
& $v_{4}$ & $560^{2}$ & 300 & 8 \\
& $v_{2}$ & $473^{3}$ & 45 & 8 \\
& & & & \\
$\mathrm{E}_{1 \mathrm{u}}$ & $v_{3}$ & $1094^{1}$ & 225 & 8 \\
& $v_{3}$ & $1034^{1}$ & 630 & 8 \\
& $v_{1}$ & $962^{2}$ & 90 & 8 \\
& $v_{4}$ & $601^{2}$ & 215 & 8 \\
& $v_{4}$ & $575^{2}$ & 120 & 8 \\
\hline
\end{tabular}

${ }^{1}$ Adams and Gardner (1974) ${ }^{2}$ Klee (1970) ${ }^{3}$ Leroy et al. (2000) ${ }^{4}$ Balan et al. (2011) 